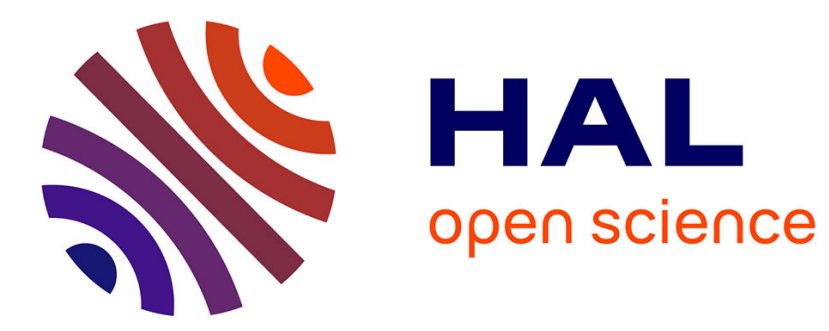

\title{
Kinematic Indices of rotation-floating space robots for on-orbit servicing
}

\author{
Mathieu Rognant, Sofiane Kraiem, Jurek Sasiadek
}

\section{To cite this version:}

Mathieu Rognant, Sofiane Kraiem, Jurek Sasiadek. Kinematic Indices of rotation-floating space robots for on-orbit servicing. 15 th IFToMM World Congress, Jun 2019, CRACOVIE, Poland. hal-02355195

\section{HAL Id: hal-02355195 \\ https://hal.science/hal-02355195}

Submitted on 8 Nov 2019

HAL is a multi-disciplinary open access archive for the deposit and dissemination of scientific research documents, whether they are published or not. The documents may come from teaching and research institutions in France or abroad, or from public or private research centers.
L'archive ouverte pluridisciplinaire HAL, est destinée au dépôt et à la diffusion de documents scientifiques de niveau recherche, publiés ou non, émanant des établissements d'enseignement et de recherche français ou étrangers, des laboratoires publics ou privés. 


\title{
Kinematic Indices of rotation-floating space robots for on-orbit servicing
}

\author{
Mathieu Rognant ${ }^{1}$, Sofiane Kraiem ${ }^{2}$, and Jurek Sasiadek ${ }^{3}$ \\ 1 mathieu.rognant@onera.fr, ONERA, Toulouse, France; \\ 2 sofiane.kraiem@isae-supaero.fr, ISAE SUPAERO, Toulouse, France; \\ 3 jurek.sasiadek@carleton.ca, Carleton University, Ottawa, Canada.
}

\begin{abstract}
There is a growing need for space and in-orbit operations that would require use of advance robotic systems. The robotics systems could be used in debris removal from orbits, as well as, in on-orbit servicing activities. This paper is addressing design and control problems related to autonomous spacecraft-manipulator system for space operation. The dynamics equations for rotation floating manipulator were introduced using Lagrange approach with additional states representing the kinetic moment exchange actuators. In this paper, a serial-link manipulator with multi degree of freedoms mounted on the satellite platform was used. For detailed analysis of base motion and manipulability of the end effector, the special indices were introduced. Simulation examples to illustrate kinematic indices were shown with physical parameters for a microsatellite from Myriade series equipped with a robotic arm.
\end{abstract}

Keywords: Space robot, On-orbit servicing, Kinematic indices

\section{Introduction}

The orbit removal and servicing topics are expected to play a key part in future space missions [1], and the studied concepts often include robotic manipulators to carry out these missions[2]. Robotic manipulators in space are already in use but are teleoperated given the complexity to steer them. Umanned servicing missions have not been perform yet and fully autonomous control of spacecraftmanipulator is still an open research area, specially with non-cooperative target.

In litterature two control modes of the spacecraft-manipulator are commonly considered: the free-flying mode where manipulator's joints and spacecraft's attitude and position are actively controlled [3]; and the free-floating mode where only manipulator's joints are controlled [4]. More recently Wilde and al. proposed in [5] to distinct three additional modes to fully covering all possible spacecraft maneuvers: rotation flying and translation flying modes which are sub-modes of the free-flying and require reaction-jet thrusters. These actuators allow to actively control the angular and/or linear momentum of the system but consume fuel and their using shorten the lifespan of the spacecraft. The third proposed mode, (rotation floating), is an extension of the free-floating mode in which the attitude of the satellite is actively controlled by using kinetic moment exchange 
devices. In this case the total kinetic momentum of the system are conserved and the used actuators (reaction wheels or control-moment gyrosocopes) require only electric power, which does not restrict the life of the spacecraft.

The free-floating manipulator kinematic was well known [6][7], and studies on space manipulator which consider kinetic moment exchange devices are focussed on the compensation of the reaction manipulator torque on the base [8][9][10].Indeed as satellites integrate such devices to achieve attitude control, these actuators are most of the time controlled separately from the joint manipulator [11] and only few address the issue of the angular momentum distribution of the system[12] and its implication to the base actuators sizing[13].

In this paper, a common control of joint and kinetic moment exchange devices is considered to achieve the desired motion of the spacecraft base and manipulator end-effector. To address design and control issues and evaluate the interest of a common control, we propose some kinematic indices which hightlight the contribution of each actuator on the controlled states.

This paper is divided as follows: first, the kinematics and dynamics for a rotation-floating robot are presented in Sec. II; then, in Sec. III, kinematic sensitivity indices are derived and their meanings are discussed; finally Sec IV outlines a simulation example which serves to illustrate the contribution of the proposed indices.

\section{Equation of motion of Rotation-floating space robots}

\section{$2.1 \quad$ Kinematic}

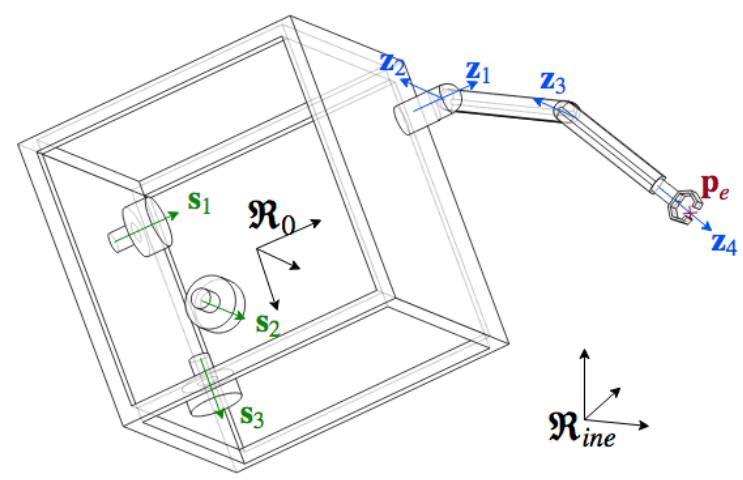

Fig. 1. Notation for a spacecraft with three reaction wheels and 4-DOFs manipulator

In this paper, Denavit-Hartenberg description is used [14] to express the end-effector position ${ }^{0} \mathbf{p}_{e}$ according to the joint configuration vector $\mathbf{q}$, and the 
end-effector relative speed to the spacecraft base reference frame $\Re_{0}$ :

$$
\left[\begin{array}{l}
0 \\
{ }^{0} \mathbf{v}_{e} \\
{ }^{0} \omega_{e}
\end{array}\right]=\mathbf{J}_{m} \dot{\mathbf{q}}
$$

where $\dot{\mathbf{q}}$ is the vector of the joint velocity $\left(\dot{\mathbf{q}}=\left[\dot{q}_{1} \cdots \dot{q}_{n}\right]^{T}\right)$ and $\mathbf{J}_{m}$ the manipulator jacobian matrix which is defined in $\mathbb{R}^{6 \times n}[7]$.

As the base of the spacecraft is also moving the total kinematic equation of the end-effector of the space robot according to inertial reference frame $\Re_{\text {ine }}$ expressed in the base $\mathcal{B}_{0}$ is

$$
\left[\begin{array}{c}
\mathbf{v}_{e} \\
\boldsymbol{\omega}_{e}
\end{array}\right]=\mathbf{J}_{b} \dot{\mathbf{x}}_{0}+\mathbf{J}_{m} \dot{\mathbf{q}}
$$

where $\dot{\mathbf{x}}_{0}$ is the vector of the linear and angular velocity according to inertial reference frame $\Re_{\text {ine }}$ expressed in the base $\mathcal{B}_{0}\left(\dot{\mathbf{x}}_{0}=\left[\begin{array}{ll}\mathbf{v}_{0}^{T} & \boldsymbol{\omega}_{0}^{T}\end{array}\right]^{T}\right)$, and $\mathbf{J}_{b} \in \mathbb{R}^{6 \times 6}$ is the base jacobian matrix [7].

\subsection{Dynamics equation of motion}

The coupled dynamics of a spacecraft-manipulator system have been extensively studied in the available literature [15][16]. To address the issue of control of rotation-floating robots, we propose to extend the free-floating dynamic model detailed in [5] by adding the states of the kinetic moment exchange actuators. By using the Lagragian approach, we just need to express the kinetic energy of the attitude actuator and add the term obtained by derivation of its kinetic energy according to the system state and time. In this paper only simple case with reaction wheels will be considered, but this approach can be easily extended to control-moment gyro. The kinetic energy $T_{r_{j}}$ of a reaction wheel of index $j$ is:

$$
T_{r_{j}}=\frac{1}{2}\left(\boldsymbol{\omega}_{0}^{T}+\omega_{r_{j}}{ }^{0} \mathbf{s}_{j}{ }^{T}\right) \mathbf{H}_{r_{j}}\left(\boldsymbol{\omega}_{0}+\omega_{r_{j}}{ }^{0} \mathbf{s}_{j}\right)+\frac{1}{2} m_{r_{j}} \dot{\mathbf{p}}_{0}^{T} \dot{\mathbf{p}}_{0}
$$

where $\omega_{r_{j}}$ is the angular velocity and ${ }^{0} \mathbf{s}_{j}$ the rotation axis expressed in $\mathcal{B}_{0} \cdot m_{r_{j}}$ and $\mathbf{H}_{r_{j}}$ are respectively the mass and the inertia matrix of the reaction wheels expressed in $\mathcal{B}_{0}$.

In this paper, one serial manipulator with $n$-DOF is mounted on the satellite. However, multiple manipulators and manipulator with complex kinematic could be considered as well [5]. Consequently, Lagrange equation derivation of (3) leads to the following dynamics equation of motion for spacecraft with $n_{r}$ reaction wheel:

$$
\left[\begin{array}{ccc}
\mathbf{H}_{0} & \mathbf{H}_{0 m} & \mathbf{H}_{0 r} \\
\mathbf{H}_{0 m}^{T} & \mathbf{H}_{m} & \mathbf{0} \\
\mathbf{H}_{0 r}^{T} & \mathbf{0} & \mathbf{H}_{r}
\end{array}\right]\left[\begin{array}{c}
\ddot{\mathbf{x}}_{0} \\
\ddot{\mathbf{q}} \\
\dot{\boldsymbol{\omega}}_{r}
\end{array}\right]+\left[\begin{array}{c}
\mathbf{c}_{0} \\
\mathbf{c}_{m} \\
\mathbf{c}_{r}
\end{array}\right]=\left[\begin{array}{c}
\mathbf{0} \\
\boldsymbol{\tau}_{m} \\
\boldsymbol{\tau}_{r}
\end{array}\right]
$$

The symbols used in this formulation are defined as follows:

$\mathbf{H}_{0} \in \mathbb{R}^{6 \times 6}$ : inertia matrix for the base. 
$\mathbf{H}_{m} \in \mathbb{R}^{n \times n}$ : inertia matrix for the links.

$\mathbf{H}_{0 m} \in \mathbb{R}^{6 \times n}$ : coupling inertia matrix between the base and the manipulator.

$\mathbf{H}_{r} \in \mathbb{R}^{n_{r} \times n_{r}}$ : inertia matrix for the reaction wheels.

$\mathbf{H}_{0 r} \in \mathbb{R}^{6 \times n_{r}}$ : coupling inertia matrix between the base and the reaction wheels. $\mathbf{c}_{0} \in \mathbb{R}^{6 \times 1}, \mathbf{c}_{m} \in \mathbb{R}^{n \times 1}$ and $\mathbf{c}_{r} \in \mathbb{R}^{n_{r} \times 1}:$ are velocity dependent non-linear terms. $\boldsymbol{\tau}_{m} \in \mathbb{R}^{n \times 1}$ and $\boldsymbol{\tau}_{r} \in \mathbb{R}^{n_{r} \times 1}$ : are manipulator joint and reaction wheels torques.

$\mathbf{H}_{0 r}{ }^{T}=\left[\mathbf{0}_{n_{r} \times 3} \mathbf{H}_{\omega r}{ }^{T}\right]$, and $\mathbf{H}_{r}$ is a $n_{r} \times n_{r}$ diagonal matrix whose the $\mathrm{i}^{\text {th }}$ component is equal to ${ }^{0} \mathbf{s}_{i}^{T} \mathbf{H}_{r_{i}}{ }^{0} \mathbf{s}_{i}$. The $\mathbf{H}_{\omega r}$ matrix is defined by:

$$
\mathbf{H}_{\omega r}=\left[\mathbf{H}_{r_{1}}{ }^{0} \mathbf{s}_{1} \cdots \mathbf{H}_{r_{n_{r}}}{ }^{0} \mathbf{s}_{n_{r}}\right]
$$

\subsection{Generalized Jacobian}

Based on the assumptions that the torques caused by the environment are negligible and the initial kinematic momentum of the spacecraft is null, the conservation of linear momentum and the angular momentum of the system yields to the generalized jacobian of the end-effector motion [4]. In this paper this expression are extended in order to discern the contribution of base actuator and the joint actuator.

$$
\mathbf{H}_{0} \dot{\mathbf{x}}_{0}+\mathbf{H}_{0 m} \dot{\mathbf{q}}+\mathbf{H}_{0 r} \boldsymbol{\omega}_{r}=\mathbf{0}
$$

The inertia matrix $\mathbf{H}_{0}$ is symetric and positive-defined then

$$
\dot{\mathbf{x}}_{0}=-\mathbf{H}_{0}^{-1} \mathbf{H}_{0 m} \dot{\mathbf{q}}-\mathbf{H}_{0}^{-1} \mathbf{H}_{0 r} \boldsymbol{\omega}_{r}
$$

From (7) one can express jacobian matrices $\mathbf{J}_{q}^{0} \in \mathbb{R}^{6 \times n}$ and $\mathbf{J}_{r}^{0} \in \mathbb{R}^{6 \times n_{r}}$ which link respectively the base kinematic to the joint and reaction wheel velocities:

$$
\mathbf{J}_{q}^{0}=-\mathbf{H}_{0}^{-1} \mathbf{H}_{0 m} \text { and } \mathbf{J}_{r}^{0}=-\mathbf{H}_{0}^{-1} \mathbf{H}_{0 r}
$$

By considering equation (2), one can also link the end-effector motion to the joint and reaction wheel velocities:

$$
\dot{\mathbf{x}}_{e}=\left(\mathbf{J}_{m}-\mathbf{J}_{b} \mathbf{H}_{0}^{-1} \mathbf{H}_{0 m}\right) \dot{\mathbf{q}}-\mathbf{J}_{b} \mathbf{H}_{0}^{-1} \mathbf{H}_{0 r} \boldsymbol{\omega}_{r}
$$

and define the jacobian matrices $\mathbf{J}_{q}^{e} \in \mathbb{R}^{6 \times n}$ and $\mathbf{J}_{r}^{e} \in \mathbb{R}^{6 \times n_{r}}$ :

$$
\mathbf{J}_{q}^{e}=\left(\mathbf{J}_{m}-\mathbf{J}_{b} \mathbf{H}_{0}^{-1} \mathbf{H}_{0 m}\right) \text { and } \mathbf{J}_{r}^{e}=-\mathbf{J}_{b} \mathbf{H}_{0}^{-1} \mathbf{H}_{0 r}
$$

\section{Kinematic Indices}

According to these models we propose in this section to analyze the participation of each actuators on the base motion. Then we evaluate the end-effector manipulability variation according to the reaction wheel control mode. 


\subsection{Base kinematic indices}

The equation (6) can be decomposed as follows:

$$
\left[\begin{array}{cc}
m_{t t} \mathbf{I}_{3} & -m_{t t}^{0} \mathbf{p}_{g}^{\times} \\
m_{t t}^{0} \mathbf{p}_{g}^{\times} & \mathbf{H}_{\omega}
\end{array}\right]\left[\begin{array}{c}
\dot{\mathbf{p}}_{0} \\
\boldsymbol{\omega}_{0}
\end{array}\right]+\left[\begin{array}{c}
\mathbf{H}_{v m} \\
\mathbf{H}_{\omega m}
\end{array}\right] \dot{\mathbf{q}}+\left[\begin{array}{c}
\mathbf{0} \\
\mathbf{H}_{\omega r}
\end{array}\right] \boldsymbol{\omega}_{r}=\left[\begin{array}{l}
\mathbf{0} \\
\mathbf{0}
\end{array}\right]
$$

where ${ }^{0} \mathbf{p}_{g}^{\times}$is the skew-symmetric matrix of ${ }^{0} \mathbf{p}_{g}$ which is the vector of the system center of gravity expressed in $\mathcal{B}_{0} ; m_{t t}$ and $\mathbf{H}_{\omega}$ are respectively the total mass of the system and the inertia matrix expressed in $\mathcal{B}_{0}$; and $\mathbf{H}_{v m}$ and $\mathbf{H}_{\omega m}$ are sub-matrices of the coupling inertia matrix $\mathbf{H}_{0 m}$ introduced in (4).

Based on this decomposition of the spacecraft base inertia matrix $\mathbf{H}_{0}$, this relation can be inversed in analytic way by using a the Banachiewicz inversion formula [17] which yields to the following expression of $\mathbf{H}_{0}^{-1}$ as detailed in [5]:

$$
\mathbf{H}_{0}^{-1}=\left[\begin{array}{cc}
\frac{\mathbf{I}_{3}}{m_{t t}}-\left({ }^{0} \mathbf{p}_{g}^{\times}\right) \mathbf{S}_{U}^{-1}\left({ }^{0} \mathbf{p}_{g}^{\times}\right) & \left({ }^{0} \mathbf{p}_{g}^{\times}\right) \mathbf{S}_{U}^{-1} \\
\mathbf{S}_{U}^{-1}\left({ }^{0} \mathbf{p}_{g}^{\times}\right) & \mathbf{S}_{U}^{-1}
\end{array}\right] \text { with } \mathbf{S}_{U}=\mathbf{H}_{\omega}+m_{t t}\left({ }^{0} \mathbf{p}_{g}^{\times}\right)\left({ }^{0} \mathbf{p}_{g}^{\times}\right)
$$

One can deduce from (8) and (12), the expression of the linear and angular part of the jacobian matrices $\mathbf{J}_{q}^{0}$ and $\mathbf{J}_{r}^{0}$ :

$$
\begin{aligned}
& \mathbf{J}_{q}^{v_{0}}=-\left(\left(\frac{\mathbf{I}_{3}}{m_{t t}}-\left({ }^{0} \mathbf{p}_{g}^{\times}\right) \mathbf{S}_{U}^{-1}\left({ }^{0} \mathbf{p}_{g}^{\times}\right)\right) \mathbf{H}_{v m}+\left({ }^{0} \mathbf{p}_{g}^{\times}\right) \mathbf{S}_{U}^{-1} \mathbf{H}_{\omega m}\right) \\
& \text { and } \begin{array}{r}
\mathbf{J}_{r}^{v_{0}}=-\left({ }^{0} \mathbf{p}_{g}^{\times}\right) \mathbf{S}_{U}^{-1} \mathbf{H}_{\omega r} \\
\mathbf{J}_{q}^{\omega_{0}}=-\left(\mathbf{S}_{U}^{-1}\left({ }^{0} \mathbf{p}_{g}^{\times}\right) \mathbf{H}_{v m}+\mathbf{S}_{U}^{-1} \mathbf{H}_{\omega m}\right) \text { and } \mathbf{J}_{r}^{\omega_{0}}=-\mathbf{S}_{U}^{-1} \mathbf{H}_{\omega r}
\end{array}
\end{aligned}
$$

An equivalent decomposition of the translation and rotation have been proposed by $\mathrm{Xu}$ in [7] to compute Joint-to-Base Coupling Factors. In order to deal with the range variation of each actuators, the following scaled jacobians will be used for analysis:

$$
\mathbf{J}_{v_{0}}=\left[\begin{array}{ll}
\mathbf{J}_{q}^{v_{0}} \mathbf{Q}_{\max } & \mathbf{J}_{r}^{v_{0}} \boldsymbol{\Omega}_{\max }
\end{array}\right] \text { and } \mathbf{J}_{\omega_{0}}=\left[\begin{array}{ll}
\mathbf{J}_{q}^{\omega_{0}} \mathbf{Q}_{\max } & \mathbf{J}_{r}^{\omega_{0}} \boldsymbol{\Omega}_{\max }
\end{array}\right]
$$

where $\boldsymbol{\Omega}_{\max }$ and $\mathbf{Q}_{\max }$ are diagonal matrices of the maximal reaction wheels and joints angular speed.

By considering relation (14), one can notice that the $\mathbf{J}_{q}^{\omega_{0}}$ and $\mathbf{J}_{r}^{\omega_{0}}$ are both factorized by $\mathbf{S}_{U}^{-1}$. That implies that the relative contributions of the joints and reaction wheels to the base angular motion do not depend on the base inertia but only on the relative angular momentum capabilities of the manipulator and the base spacecraft actuator. This highlights that the distribution of the angular momentum is a critical point to insure the controlability of the system and dedicated control algorithms are required [12].

A dedicated analysis of the components of $\mathbf{S}_{U} \mathbf{J}_{\omega_{0}}$ matrix of the system in a design phasis could address this issue. The sum of the reaction wheels angular momentum is bounded by $\left\|\mathbf{S}_{U} \mathbf{J}_{r}^{\omega_{0}} \boldsymbol{\Omega}_{\max }\right\|$. Thus, in the angular momemtum space, this sum belongs to the invariant polyedron whose the faces are normal to the $\mathbf{s}_{j}$ axis. Concerning the manipulator, its angular momentum is bounded by 
$\left\|\mathbf{S}_{U} \mathbf{J}_{q}^{\omega_{0}} \boldsymbol{\Omega}_{\text {max }}\right\|$, which is strongly sensitive to the joint configuration (variation of the gravity center position and manipulator inertia (14)). Thus, to distinguish the joint and reaction wheel contributions to the base motion according to the joint configuration, we proposed the indices $\kappa_{\omega_{0}}^{q}$ and $\kappa_{\omega_{0}}^{r}$ defined as follow:

$$
\kappa_{\omega_{0}}^{q}=\frac{\left\|\mathbf{S}_{U} \mathbf{J}_{q}^{\omega_{0}} \mathbf{Q}_{\max }\right\|_{F}^{2}}{\left\|\mathbf{S}_{U} \mathbf{J}_{\omega_{0}}\right\|_{F}^{2}} ; \kappa_{\omega_{0}}^{r}=\frac{\left\|\mathbf{S}_{U} \mathbf{J}_{r}^{\omega_{0}} \boldsymbol{\Omega}_{\max }\right\|_{F}^{2}}{\left\|\mathbf{S}_{U} \mathbf{J}_{\omega_{0}}\right\|_{F}^{2}}
$$

where $\|\cdot\|_{F}$ is the Frobenius norm. As this norm can be evaluated analytically, these indicators could be an interesting tool to caracterize the base controlability on pre-design stages or select preferential joint configuration for control purpose. For example, joint configurations whose the index $\kappa_{\omega_{0}}^{q}$ respect the constraint $\kappa_{\omega_{0}}^{q} \leq 0.5$ are more suitable to achieve motion without changing the base attitude.

\subsection{End-effector indices}

The end-effector control motion is the highest priority task, however the dynamic coupling with the motion base could significantly reduce the manipulator capability, specially when fixed base attitude is required. To analyse this point we propose in this section dedicated manipulabity indices.

As for the base, one can defined from (10) the scaled jacobians of the linear and angular motion of the end-effector:

$$
\mathbf{J}_{v_{e}}=\left[\begin{array}{ll}
\mathbf{J}_{q}^{v_{e}} \mathbf{Q}_{\max } & \mathbf{J}_{r}^{v_{e}} \boldsymbol{\Omega}_{\max }
\end{array}\right] \text { and } \mathbf{J}_{\omega_{e}}=\left[\begin{array}{ll}
\mathbf{J}_{q}^{\omega_{e}} \mathbf{Q}_{\max } & \mathbf{J}_{r}^{\omega_{e}} \boldsymbol{\Omega}_{\max }
\end{array}\right]
$$

Then, the end-effector manipulability can be caracterized by the operator $\mu(\mathbf{J})=$ $\sqrt{\operatorname{det}\left(\mathbf{J J}^{T}\right)}[6]$

$$
\mu_{v_{e}}=\mu\left(\mathbf{J}_{v_{e}}\right) \text { and } \mu_{\omega_{e}}=\mu\left(\mathbf{J}_{\omega_{e}}\right)
$$

As proposed by Nenchev and al. in [18] for redundant manipulator, null motion projector could be used to restrict the joint motion to a sub-space which insure zero base attitude disturbance. In the same manner null motion projector could be defined for a common control of the reaction-wheel and joint velocities as follows:

$$
\mathbf{N}_{\omega_{0}}=\mathbf{I}-\mathbf{J}_{\omega_{0}}^{\dagger} \mathbf{J}_{\omega_{0}}
$$

where $\mathbf{J}_{\omega_{0}}^{\dagger}$ is the pseudo-inverse of the $\mathbf{J}_{\omega_{0}}$. By using this projector, one can define manipulability indices of the end-effector without base motions:

$$
\mu_{v_{e}}^{n}=\mu\left(\mathbf{J}_{v_{e}} \mathbf{N}_{\omega_{0}}\right) \text { and } \mu_{\omega_{e}}^{n}=\mu\left(\mathbf{J}_{\omega_{e}} \mathbf{N}_{\omega_{0}}\right)
$$

\section{Simulation Example}

In this section numerical simulations are provided to illustrate the kinematic indices proposed in this paper. All these evalutions are carried out by using the physical parameters of the first microsatellite in the Myriade series, Demeter 
[19]. Demeter is equipped with three reaction wheels, which nominaly present an inertia of $4.10^{-4} \mathrm{~kg} \cdot \mathrm{m}^{2}$ on their main axis and a maximal angular speed of $2800 \mathrm{rpm}$. Concerning the manipulator, the kinematic chain represented in figure 1 will be used. As this study is focussed on the end-effector linear speed, only the 3 first DOFs will be considered. As current space manipulator such as Canadarm2 and JEMRMS do not exceed the $0.7 \mathrm{rpm}$ [20] we assume that joints angular speed should not overpass $0.5 \mathrm{rpm}$ and the used links mass, dimension and inertia are summarized in Table 1 . In a context of pre-design, the sizing of

\begin{tabular}{|c|c|c|c|c|c|}
\hline- & masse $(\mathrm{kg})$ & dimension $(\mathrm{m})$ & $\mathrm{Ixx}\left(\mathrm{kg} . \mathrm{m}^{2}\right)$ & Iyy $\left(\mathrm{kg} . \mathrm{m}^{2}\right)$ & Izz $\left._{\left(\mathrm{kg} . \mathrm{m}^{2}\right.}\right)$ \\
\hline base & 100 & $0.6 \times 0.6 \times 0.6$ & 40 & 20 & 40 \\
\hline link 1 & 1.97 & 0.15 & $35.10^{-4}$ & $7.10^{-4}$ & $4.10^{-4}$ \\
\hline link 2 & 1.58 & 1 & $28.10^{-4}$ & $917.10^{-4}$ & $268.10^{-4}$ \\
\hline link 3 & 1.17 & 0.6 & $7.10^{-4}$ & $924.10^{-4}$ & $273.10^{-4}$ \\
\hline
\end{tabular}

Table 1. Model parameters

the spacecraft actuators is a critical task. Therefore, in this example, we proposed using the suggested indices to compare two configurations of reaction wheels: a first one equivalent of Demeter's and a second one with twice the nominal inertia $\mathbf{H}_{r}$.

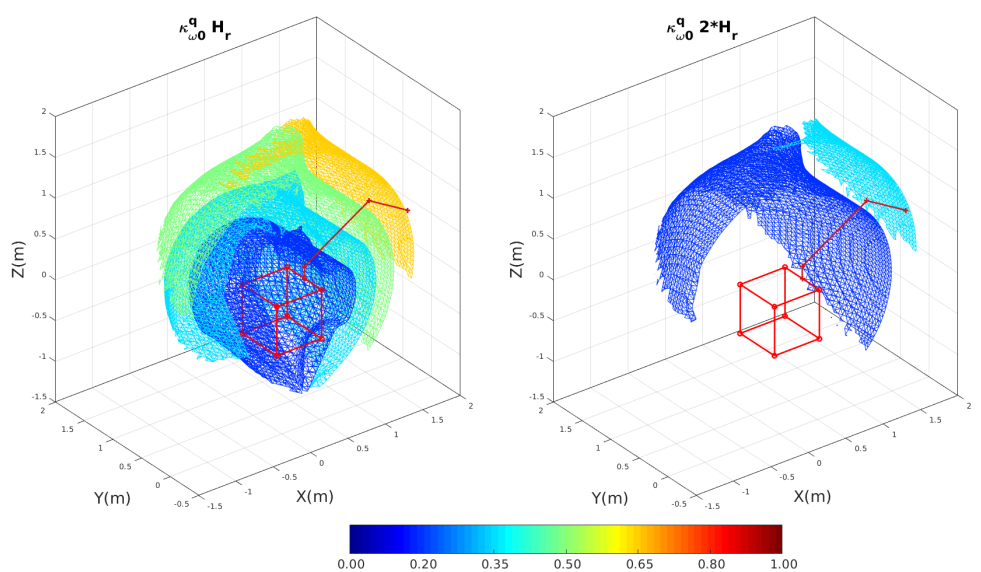

Fig. 2. Variation of the joints contribution to the base motion $\left(\kappa_{\omega_{0}}^{q}\right)$ plotted in $\Re_{0}$ for the initial reaction wheel (left) and the second actuators configuration (right) 


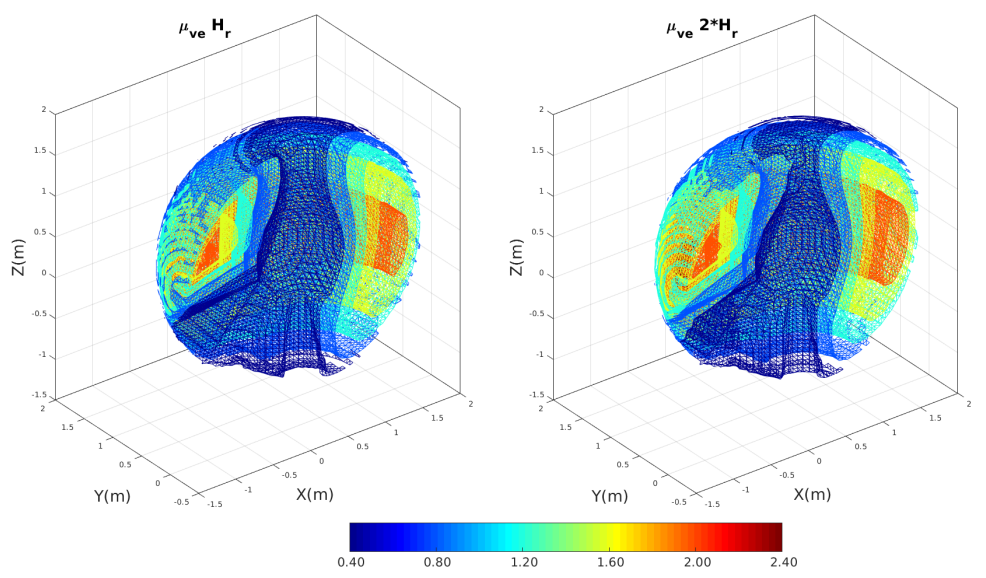

Fig. 3. Evolution of the end-effector normalized manipulability indices plotted in $\Re_{0}$ for the initial reaction wheel (left) and the second actuators configuration (right)

To achieve this analysis, the indices have been evaluated for the two configurations in 6859 joints configurations uniformly distributed in the half-workspace of the manipulator because the system is symmetric about the XZ plane. In figures 2, 3 and 4 iso-surfaces of these indices are plotted and colours reflect value. In figure 3 and 4 manipulability indices are normalized by the mean manipulability of the manipulator on a fixe base. Figure 2 points that increasing the reaction wheels inertia allows to decrease the contribution of the joints and respectively to raise the one of the reaction wheels on the base attitude motion, as $\kappa_{\omega_{0}}^{q}$ and $\kappa_{\omega_{0}}^{r}$ are complementary. One can also note that, the joint configurations which involve the most the base motion are those where the end-effector is farthest from the system center of mass. In these critical configurations, doubling the inertia of the reaction wheels allow to reduce the joints impact on the base from 0.65 to 0.4 and to ensure a stable attitude without using thrusters. However, the manipulability of $\mathbf{v}_{e}$, represented in figure 3 , increases a less than 0.1 if the base is able to move during the control of the end-effector. This is due to the small ratio between the maximal spacecraft angular speed and joints angular speed considered in our example. In this case, reducing the joint impact to the base with bigger reaction wheel inertia does not affect the manipulability of $\boldsymbol{v}_{\boldsymbol{e}}$. Nevertheless, when null-motion is used, figure 4 highlights an improvement of the manipulability of $\mathbf{v}_{e}$ with more angular momentum in reaction wheels. As underlined by the figure 2 , the second configuration presents a better momentum repartition for this type of control. 


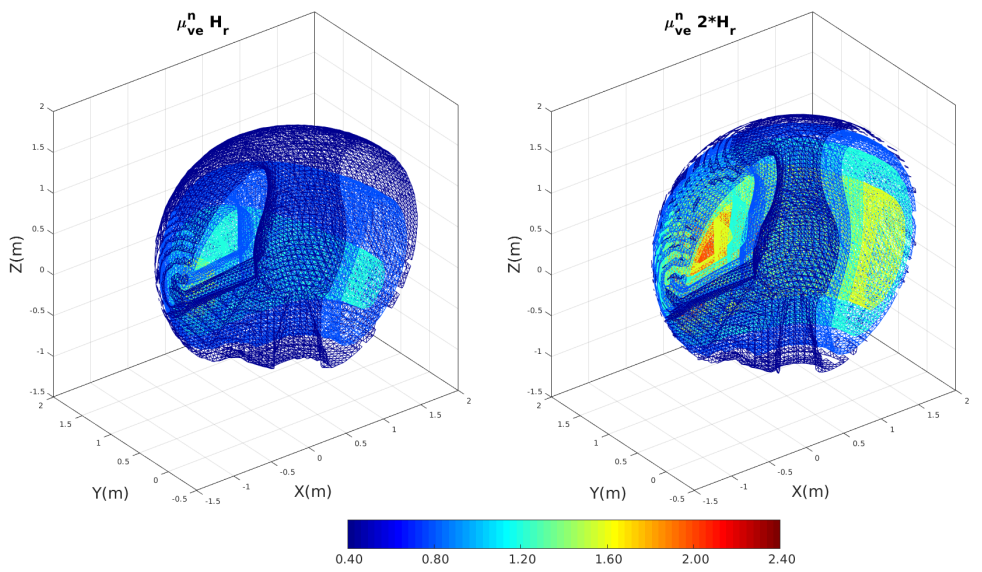

Fig. 4. Evolution of the end-effector normalized manipulability indices with a fix base attitude constraint plotted in $\Re_{0}$ for the initial reaction wheel (left) and the second actuators configuration (right)

\section{Conclusion}

This paper presents some aspects of control for rotation-floating space robot for on-orbit servicing. Special indices for analyzing dynamic capabilities of the robot were suggested and used in simulation experiments. Simulation examples to illustrate kinematic indices were shown with physical parameters for a microsatellite from Myriade series Demeter. This paper compared two configurations of spacecraft actuators: the first, equivalent to Demeter configuration and the second with twice as large, the nominal inertia. It was shown that increasing the reaction wheels inertia allows to reduce the contribution of the joints and raises respective wheels contribution to the motion of robotic spacecraft. Also, it could be noted that the manipulability of the arm increases, if the base is able to move during the end-effector control. The use of suggested indicators allowed to evaluate a system and determine momentum distribution between manipulator and the base of spacecraft.

\section{References}

1. Long, A., Richards, M., Hastings, D.E.: On-orbit servicing: a new value proposition for satellite design and operation. Journal of Spacecraft and Rockets 44(4), 964976 (2007)

2. Flores-Abad, A., Ma, O., Pham, K., Ulrich, S.: A review of space robotics technologies for on-orbit servicing. Progress in Aerospace Sciences 68, 1-26 (2014)

3. Shibli, M.: Unified modeling approach of kinematics, dynamics and control of a free-flying space robot interacting with a target satellite. Intelligent Control and Automation 2(01), 8 (2011) 
4. Yoshida, K., Sashida, N., Kurazume, R., Umetani, Y.: Modeling of collision dynamics for space free-floating links with extended generalized inertia tensor. In: Robotics and Automation, 1992. Proceedings., 1992 IEEE International Conference on, pp. 899-904. IEEE (1992)

5. Wilde, M., Kwok Choon, S., Grompone, A., Romano, M.: Equations of motion of free-floating spacecraft-manipulator systems: An astronautical engineers tutorial. Frontiers in Robotics and AI 5, 41 (2018)

6. Umetani, Y., Yoshida, K.: Workspace and manipulability analysis of space manipulator. Transactions of the Society of Instrument and Control Engineers 26(2), 188-195 (1990)

7. Xu, W., Peng, J., Liang, B., Mu, Z.: Hybrid modeling and analysis method for dynamic coupling of space robots. IEEE Transactions on Aerospace and Electronic Systems 52(1), 85-98 (2016)

8. Ellery, A.: Robotic in-orbit servicers the need for control moment gyroscopes for attitude control. The Aeronautical Journal 108(1082), 207-214 (2004)

9. Dimitrov, D.N., Yoshida, K.: Utilization of distributed momentum control for planning approaching trajectories of a space manipulator to a target satellite. In: Proceedings of the 8th Int. Symposium on Artificial Intelligence, Robotics and Automation in Space, Munich, Germany (2005)

10. Wu, Y., Han, F., Zheng, M., He, M., Chen, Z., Hua, B., Wang, F.: Attitude control for on-orbit servicing spacecraft using hybrid actuator. Advances in Space Research 61(6), 1600-1616 (2018)

11. Jaekel, S., Lampariello, R., Rackl, W., De Stefano, M., Giordano, A.M., Porges, O., Pietras, M., Oumer, N., Brunner, B., Ratti, J., et al.: Design and operational elements of the robotic subsystem for the e. deorbit debris removal mission. Frontiers in Robotics and AI 5, 100 (2018)

12. Oki, T., Nakanishi, H., Yoshida, K.: Time-optimal manipulator control for management of angular momentum distribution during the capture of a tumbling target. Advanced Robotics 24(3), 441-466 (2010)

13. Kim, Y.M., Kim, B.K.: Energy-efficient trajectory generation for space manipulators with reaction wheels under a fixed base orientation. Journal of Intelligent \& Robotic Systems 76(2), 219-237 (2014)

14. Khalil, W., Dombre, E.: Modelisation, identification and control of robots (2002)

15. Dubowsky, S., Papadopoulos, E.: The kinematics, dynamics, and control of freeflying and free-floating space robotic systems. IEEE Transactions on robotics and automation 9(5), 531-543 (1993)

16. Moosavian, S.A.A., Papadopoulos, E.: Free-flying robots in space: an overview of dynamics modeling, planning and control. Robotica 25(5), 537-547 (2007)

17. Baksalary, J.K., Styan, G.P.: Generalized inverses of partitioned matrices in banachiewicz - schur form. Linear algebra and its applications 354(1-3), 41-47 (2002)

18. Nenchev, D.N., Yoshida, K., Vichitkulsawat, P., Uchiyama, M.: Reaction null-space control of flexible structure mounted manipulator systems. IEEE Transactions on Robotics and Automation 15(6), 1011-1023 (1999)

19. Pittet, C., Arzelier, D.: Demeter: A benchmark for robust analysis and control of the attitude of flexible micro satellites. IFAC Proceedings Volumes 39(9), 661-666 (2006)

20. Laryssa, P., Lindsay, E., Layi, O., Marius, O., Nara, K., Aris, L., Ed, T.: International space station robotics: a comparative study of era, jemrms and mss. In: 7th ESA Workshop on Advanced Space Technologies for Robotics and Automation, pp. 19-21 (2002) 